\title{
Análise da Eficiência Computacional para Solução do Problema da Cinemática Inversa de Robôs Seriais Utilizando a Teoria de Bases de Gröbner
}

\author{
Sérgio R. X. Silva *. Leizer Schnitman ** \\ Vitalino C. Filho *** \\ *PPGM - Programa de Pós-Graduação em Mecatrônica, Departamentos de Ciência \\ da Computação \& Engenharia Mecânica, Universidade Federal da Bahia - UFBA \\ Rua Prof. Aristides Novis 02, 40.210-630, Federação, Salvador, BA, Brasil, (e-mail: srxsilva@uneb.br). \\ ** PPGM - Programa de Pós-Graduação em Mecatrônica, Departamentos de Ciência \\ da Computação \& Engenharia Mecânica, Universidade Federal da Bahia - UFBA \\ Rua Prof. Aristides Novis 02, 40.210-630, Federação, Salvador, BA, Brasil, (e-mail: leizer@ufba.br). \\ *** Universidade Federal do Pampa - UNIPAMPA \\ Av. Pedro Anunciação 111, 96.570-000, Bairro Vila Batista, Caçapava do Sul, RS, Brasil \\ (e-mail: vitalinocesca@unipampa.edu.br).
}

\begin{abstract}
This paper presents an analysis of computational efficiency in the solution of the inverse kinematics problem of serial manipulator robots. Two approaches are investigated: the first approach uses the Paul method from the matrix obtained by the Denavit-Hartenberg algorithm, and the second approach uses Gröbner Basis Theory. With each approach, the problem of inverse kinematics for two serial robots will be solved. When comparing each method, this paper will demonstrate that the method from Gröbner Basis Theory is more computationally efficient.
\end{abstract}

Resumo: Este artigo traz uma análise da eficiência computacional na solução do problema da cinemática inversa de robôs manipuladores seriais. São investigadas duas abordagens: a primeira abordagem utiliza o método de Paul a partir da matriz obtida pelo algoritmo de Denavit-Hartenberg, e a segunda abordagem utiliza a Teoria das Bases de Gröbner. Com cada abordagem, o problema da cinemática inversa para dois robôs seriais será resolvido. Ao comparar cada método, este artigo demonstrará que o método a partir da Teoria das Bases de Gröbner é mais eficiente computacionalmente.

Keywords: Robotics; Inverse kinematic; Computational Efficiency; Paul Method; Gröbner Basis.

Palavras-chaves: Robótica; Cinemática Inversa; Eficiência Computacional; Método de Paul; Bases de Gröbner.

\section{INTRODUÇÃO}

Dois problemas clássicos na cinemática de manipuladores robóticos são a cinemática direta e a inversa. Na cinemática direta, a posição e orientação do efetuador são calculados a partir das variáveis das juntas do robô. Na cinemática inversa, os movimentos das juntas individuais são calculados a partir da posição e orientação do efetuador final do manipulador.

Paul, Shimano e Mayer (1981) utilizaram álgebra matricial para obter uma solução para o problema da cinemática inversa de um robô manipulador. Lee (1982) e Yih e Youm (1988) utilizaram uma abordagem geométrica para resolver este problema. Posteriormente, Huang e Milenkovic (1983) e Manseur e Doty (1988) desenvolveram procedimentos iterativos para se obter uma solução para o problema cinemático inverso.

Jacques Denavit e Richard Hartenberg introduziram uma convenção com o intuito de padronizar as coordenadas de sistemas de referência para ligações espaciais (Denavit e
Hartenberg, 1955); após uma década, os mesmos autores apresentaram o algoritmo para solução da cinemática de sistemas articulados (Hartenberg e Denavit, 1965). A demonstração da importância deste algoritmo para a análise cinemática de sistemas robóticos foi apresentada por Paul (1981) após dezesseis anos.

Segundo Sciavicco e Siciliano (1996), quando a cinemática inversa é solucionada a partir da matriz de transformação homogênea, pode-se deparar com os seguintes problemas: a necessidade de métodos e algoritmos adicionais para resolver o problema, e cálculos matriciais complexos que requerem maiores esforços computacionais.

Levando em consideração os problemas para a solução da cinemática inversa, um método alternativo será utilizado neste artigo, o método da Teoria das Bases de Gröbner. Os dados de desempenho computacional, obtidos neste artigo, comprovarão que esta escolha reduzirá o custo computacional para a solução da cinemática inversa de manipuladores robóticos seriais. 
O método das Bases de Gröbner, desenvolvido por Bruno Buchberger em sua tese de doutorado, consiste em um algoritmo algébrico que pode ser aplicado a um dado conjunto de polinômios não nulos, produzindo um conjunto finito de geradores, de tal modo que seja possível identificar, a partir destes geradores, quando um certo polinômio pertence ao conjunto original dado (Buchberger, 2006).

Ao encontrar soluções para este conjunto de geradores, encontram-se também, soluções para todo o conjunto. Com o auxílio de um software de computação algébrica, pode-se calcular todas estas soluções, incluindo os casos não tão facilmente resolvidos utilizando álgebra matricial ou aplicações geométricas, devido à complexidade da geometria de alguns robôs manipuladores.

Com o objetivo de familiarizar o leitor com cada método, os robôs manipuladores Stäubli TS20, um robô do tipo SCARA, e o Unimation PUMA 560, um manipulador com seis juntas rotativas, serão utilizados como estudo de caso aplicando o método de Paul a partir da matriz de Denavit-Hartenberg e o método proposto neste trabalho (Bases de Gröbner) para solução do problema da cinemática inversa.

Diferentemente de Wang, Hang e Yang (2006), que também apresentaram uma solução para a cinemática inversa de um manipulador serial 6R utilizando o método das Bases de Gröbner, uma base com um número reduzido de polinômios será determinada neste artigo, uma vez que estes autores, ao utilizarem o método de solução da cinemática de mecanismos proposta por Duffy e Crane (1980), produziram uma base de Gröbner com 72 polinôminos.

Cada método será analisado e suas respectivas soluções serão comparadas com a mensuração do custo computacional.

\section{BASES DE GRÖBNER}

A Teoria de Bases de Gröbner permite que seja utilizado um algoritmo para resolver sistemas de equações polinomiais. Mais formalmente, este algoritmo, chamado algoritmo de Buchberger (Buchberger, 1995), aplica técnicas de álgebra computacional para ideais polinomiais específicos, produzindo uma base de Gröbner que pode ser usada para encontrar soluções para um conjunto de diferentes polinômios não nulos para um determinado ideal.

De acordo com Boas e Marcos (2016), resumidamente, uma base de Gröbner para um sistema de equações polinomiais é um novo sistema com as mesmas soluções, porém mais simples de ser resolvido. Essa definição justifica a escolha da Teoria de Bases de Gröbner para a solução da cinemática inversa de manipuladores seriais com o objetivo de reduzir o custo computacional.

A Teoria de Bases de Gröbner é muito aplicada em áreas distintas da Matemática. Por exemplo, Robbiano (1998), utiliza bases de Gröbner para resolver problemas de Estatística. Wang (1998), mostra como as bases de Gröbner podem ser utilizadas para solucionar problemas geométricos complexos. Cox, Little e O'Shea (1992), têm dedicado seus trabalhos para as aplicações da teoria de bases de Gröbner em Geometria, Teoria dos Grafos, e Robótica.

Segundo Sturmfels (2005), ao se determinar uma base de Gröbner, pelo menos um elemento desta base será dado em termos de uma variável no conjunto. Isso faz com que o cálculo da cinemática inversa a partir de uma base de Gröbner seja eficiente matematicamente, porque através de substituições retroativas, pode-se encontrar uma solução para o restante das variáveis do conjunto com uma redução do número de operações de ponto flutuante.

O algoritmo de Buchberger constrói uma Base de Gröbner para um ideal a partir de um conjunto finito de geradores. De acordo com Cox, Little e O'Shea (1992), para desenvolver o Algoritmo de Buchberger, faz-se necessário entender a natureza dos S-polinômios.

Tomando $0 \neq f, g \in k\left[x_{1}, \ldots, x_{n}\right]$, e considerando $L=$ $m m c(m l(f), m l(g))$. A combinação $S(f, g)=\frac{L}{t l(f)} f-$ $\frac{L}{t l(g)} g$ é chamada S-polinômio de $f$ e $g$, onde $(m m c)$ é o mínimo múltiplo comum entre os monômios líderes $(m l)$ dos polinômios; e $(t l)$, o termo líder de cada polinômio.

O S-polinômio é produzido de modo a cancelar os termos líderes dos polinômios envolvidos. $\mathrm{O}$ algoritmo de Buchberger recebe como entrada um conjunto finito $F=$ $\left\{f_{1}, f_{2}, \cdots, f_{n}\right\}$ de geradores de um ideal $I$ de $k\left\{x_{1}, \cdots, x_{n}\right\}$ e fornece um conjunto $G$, que é uma Base de Gröbner em $I$. Este algoritmo pode ser descrito como:

$$
\begin{aligned}
& G \leftarrow F \\
& \text { repita } \\
& G^{\prime} \leftarrow G \\
& \text { para cada } \operatorname{par} f_{i}, f_{j} \in G^{\prime} \text { faça } \\
& r \leftarrow \text { resto da divisão }\left(G, S\left(f_{i}, f_{j}\right)\right) \\
& \text { se } r \neq 0 \\
& \text { então } G \leftarrow G \cup\{r\}
\end{aligned}
$$

Sistemas de computação algébrica, como por exemplo: MAPLE, Singular, muMath, AXIOM, Mathematica, Macaulay, CoCoA, e Reduce, incluem pacotes para lidar com Bases de Gröbner.

\section{CINEMÁTICA INVERSA UTILIZANDO O MÉTODO DE PAUL A PARTIR DA MATRIZ DE DENAVIT- HARTENBERG}

A cinemática inversa para os robôs SCARA Stäubli TS20 e Unimation PUMA 560 será solucionada aplicando o método de Paul a partir da matriz de Denavit-Hartenberg.

\subsection{Cinemática Inversa do SCARA Stäubli TS20 a partir do método de Paul}

O robô SCARA Stäubli TS20 é normalmente utilizado para executar tarefas de linha de montagem. A escolha deste se dá por conta de sua geometria simplificada e pelo fato de o 
mesmo possuir diferentes tipos de juntas: três rotativas e uma prismática. Este manipulador robótico possui quatro graus de liberdade, no entanto, o modelo cinemático incide sobre as três primeiras juntas, uma vez que o último elo refere-se ao efetuador final do robô.

O manipulador Stäubli TS20 é representado na Fig. 1, com os seus principais sistemas de referência.

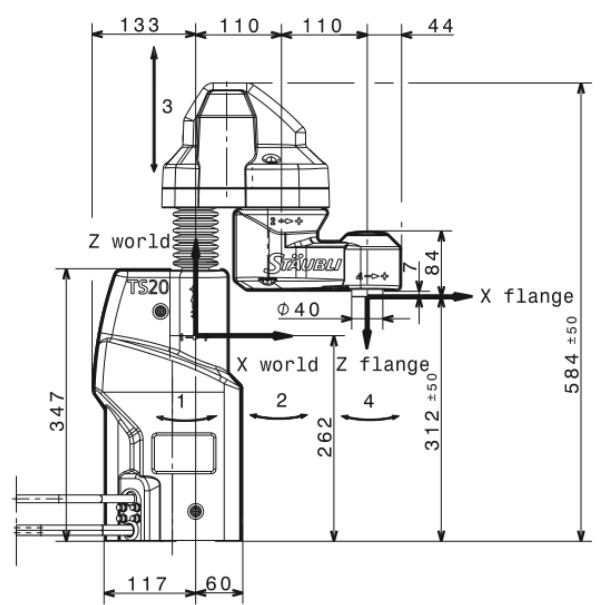

Fig. 1 Sistemas de referência do SCARA Stäubli TS20

Todos os parâmetros cinemáticos deste manipulador estão listados na Tabela 2. Onde $i$ representa o número da junta.

Tabela 1. Parâmetros cinemáticos SCARA Stäubli TS20

\begin{tabular}{|c|c|c|c|c|}
\hline$i$ & $\alpha_{i}$ (graus) & $a_{i}(\mathrm{~mm})$ & $d_{i}(\mathrm{~mm})$ & $\theta_{i}$ (graus) \\
\hline 1 & 0 & 0 & 347 & $\theta_{1}$ \\
\hline 2 & 0 & 100 & 0 & 0 \\
\hline 3 & 0 & 100 & 0 & $\theta_{3}$ \\
\hline
\end{tabular}

De acordo com Tsai (1999), na representação de DenavitHartenberg (D-H), associa-se o eixo de rotação para junta rotativa ou o eixo de translação para junta prismática ao eixo $z$ do sistema coordenado fixado na junta, e cada matriz é representada pelo produto de quatro transformações básicas envolvendo rotações $(R)$ e translações $(T r)$ como pode ser observado em (1). Utiliza-se $s_{\theta_{k}}$ para $\operatorname{sen}\left(\theta_{k}\right)$ e $c_{\theta_{k}}$ para $\cos \left(\theta_{k}\right)$.

$$
\begin{gathered}
A_{(k-1) k}=R_{z_{k}, \theta_{k}} \cdot \operatorname{Tr}_{z_{k}, d_{k}} \cdot \operatorname{Tr}_{x_{k}, a_{k}} \cdot R_{x_{k}, \alpha_{k}} \\
=\left[\begin{array}{cccc}
C_{\theta_{k}} & -s_{\theta_{k}} & 0 & 0 \\
s_{\theta_{k}} & c_{\theta_{k}} & 0 & 0 \\
0 & 0 & 1 & 0 \\
0 & 0 & 0 & 1
\end{array}\right]\left[\begin{array}{cccc}
1 & 0 & 0 & 0 \\
0 & 1 & 0 & 0 \\
0 & 0 & 1 & d_{k} \\
0 & 0 & 0 & 1
\end{array}\right] \\
{\left[\begin{array}{cccc}
1 & 0 & 0 & a_{k} \\
0 & 1 & 0 & 0 \\
0 & 0 & 1 & 0 \\
0 & 0 & 0 & 1
\end{array}\right]\left[\begin{array}{cccc}
1 & 0 & 0 & 0 \\
0 & c_{\alpha_{k}} & -s_{\alpha_{k}} & 0 \\
0 & s_{\alpha_{k}} & c_{\alpha_{k}} & 0 \\
0 & 0 & 0 & 1
\end{array}\right]}
\end{gathered}
$$

Esta operação resulta em (2), sendo que através desta, é realizado o mapeamento de coordenadas entre os elos do robô manipulador.

$$
A_{(k-1) k}=\left[\begin{array}{cccc}
c_{\theta_{k}} & -s_{\theta_{k}} c_{\alpha_{k}} & s_{\theta_{k}} s_{\alpha_{k}} & a_{k} c_{\theta_{k}} \\
s_{\theta_{k}} & c_{\theta_{k}} c_{\alpha_{k}} & -c_{\theta_{k}} s_{\alpha_{k}} & a_{k} s_{\theta_{k}} \\
0 & s_{\alpha_{k}} & c_{\alpha_{k}} & d_{k} \\
0 & 0 & 0 & 1
\end{array}\right]
$$

Multiplicando todas as matrizes de transformação obtidas pelo algoritmo de Denavit-Hartenberg, obtém-se a matriz de transformação homogênea que parte da base à extremidade do efetuador final, ou seja, variando $k$ de 0 a $n$. Esta matriz resultante pode ser considerada como a solução do problema da cinemática direta. Resumidamente, para um manipulador com $n$ graus de liberdade:

$$
T=A_{01} \cdot A_{12} \cdot \ldots \cdot A_{(n-1) n}=\left[\begin{array}{cccc}
n_{x} & o_{x} & a_{x} & x \\
n_{y} & o_{y} & a_{y} & y \\
n_{z} & o_{z} & a_{z} & z \\
0 & 0 & 0 & 1
\end{array}\right],
$$

onde cada matriz $A_{i j}$ é uma matriz D-H, definida em (2).

Cada conjunto junta/elo é descrito em termos dos quatro parâmetros cinemáticos do robô: $a_{k}, d_{k}, \alpha_{k}$ e $\theta_{k}$. Estes parâmetros, listados na Tabela 1, são utilizados para formar as três matrizes de transformação do manipulador.

Após a construção de todas as matrizes de transformação utilizando os parâmetros cinemáticos, e multiplicação das mesmas; obtém-se a matriz de transformação homogênea (4), solução do problema da cinemática direta do SCARA Stäubli TS20. Utiliza-se $s_{k}$ para $\operatorname{sen}\left(\theta_{k}\right)$ e $c_{k}$ para $\cos \left(\theta_{k}\right)$

$$
T=\left[\begin{array}{cccc}
-s_{1} s_{3}+c_{1} c_{3} & -c_{1} s_{3}-s_{1} c_{3} & 0 & 110\left(c_{1} c_{3}-s_{1} s_{3}+c_{1}\right) \\
s_{1} c_{3}+c_{1} s_{3} & -s_{1} s_{3}+c_{1} c_{3} & 0 & 110\left(s_{1} c_{3}+c_{1} s_{3}+s_{1}\right) \\
0 & 0 & 1 & 347+d_{2} \\
0 & 0 & 0 & 1
\end{array}\right]
$$

Sendo que os três primeiros elementos da última coluna da matriz representam a posição do efetuador: $x=110\left(c_{1} c_{3}-\right.$ $\left.s_{1} s_{3}+c_{1}\right), y=110\left(s_{1} c_{3}+c_{1} s_{3}+s_{1}\right)$ e $z=347+d_{2}$.

Para resolver o problema da cinemática inversa, utiliza-se o método de Paul (1981). Partindo deste algoritmo complementar, cada membro de (4) é multiplicado à esquerda por $A_{01}^{-1}$, obtendo-se (5).

$$
\begin{aligned}
& A_{01}^{-1} \cdot T=A_{01}^{-1} \cdot A_{01} \cdot A_{12} \cdot A_{23}=A_{12} \cdot A_{23} \\
& =\left[\begin{array}{cccc}
c_{1} & s_{1} & 0 & 0 \\
-s_{1} & c_{1} & 0 & 0 \\
0 & 0 & 1 & -347 \\
0 & 0 & 0 & 1
\end{array}\right]\left[\begin{array}{cccc}
n_{x} & o_{x} & a_{x} & x \\
n_{y} & o_{y} & a_{y} & y \\
n_{z} & o_{z} & a_{z} & z \\
0 & 0 & 0 & 1
\end{array}\right] \\
& =\left[\begin{array}{cccc}
c_{3} & -s_{3} & 0 & 110 c_{3}+110 \\
s_{3} & c_{3} & 0 & 110 s_{3} \\
0 & 0 & 1 & d_{2} \\
0 & 0 & 0 & 1
\end{array}\right]
\end{aligned}
$$

Efetuando a multiplicação do lado esquerdo, obtém-se (6). 


$$
\begin{array}{ccccc}
n_{x} c_{1}+n_{y} s_{1} & o_{x} c_{1}+o_{y} s_{1} & a_{x} c_{1}+a_{y} s_{1} & x c_{1}+y s_{1} \\
-n_{x} s_{1}+n_{y} c_{1} & -o_{x} s_{1}+o_{y} c_{1} & -a_{x} s_{1}+a_{y} c_{1} & -x s_{1}+y c_{1} \\
n_{z} & o_{z} & a_{z} & z-347 \\
0 & 0 & & 0 \\
0 & =\left[\begin{array}{cccc}
c_{3} & -s_{3} & 0 & 110 c_{3}+110 \\
s_{3} & c_{3} & 0 & 110 s_{3} \\
0 & 0 & 1 & d_{2} \\
0 & 0 & 0 & 1
\end{array}\right]
\end{array}
$$

Desta forma, $d_{2}=z-347$, fica determinada uma das três variáveis desconhecidas. No entanto, ainda é necessário determinar os ângulos $\theta_{1}$ e $\theta_{3}$. Elevam-se ao quadrado ambos os membros das duas equações obtidas a partir dos dois elementos da quarta coluna das matrizes. Em seguida, após algumas substituições, (7) e (8) são determinados.

$$
\begin{gathered}
\theta_{1}=\tan ^{-1}\left(\frac{y\left(x^{2}+y^{2}\right) \mp\left(\sqrt{-\left(x^{2}+y^{2}\right)\left(x^{2}+y^{2}-220^{2}\right)}\right) x}{x\left(x^{2}+y^{2}\right) \pm\left(\sqrt{-\left(x^{2}+y^{2}\right)\left(x^{2}+y^{2}-220^{2}\right)}\right) y}\right) \\
\theta_{3}=\tan ^{-1}\left( \pm \frac{\sqrt{-\left(x^{2}+y^{2}\right)\left(x^{2}+y^{2}-220^{2}\right)}}{x^{2}+y^{2}-2\left(110^{2}\right)}\right)
\end{gathered}
$$

Logo, os valores de junta $\left(\theta_{1}, d_{2}\right.$ e $\left.\theta_{3}\right)$ podem ser determinados a partir das equações deduzidas nesta seção.

\subsection{Cinemática Inversa do Unimation PUMA 560 a partir do método de Paul}

O Unimation PUMA 560 é um manipulador robótico com seis graus de liberdade e todas as juntas rotativas. Todos os parâmetros cinemáticos deste manipulador estão listados na Tabela 2. Onde $i$ representa o número da junta.

Tabela 2. Parâmetros cinemáticos do Puma 560

\begin{tabular}{|c|c|c|c|c|}
\hline$i$ & $\alpha_{i-1}$ (graus) & $a_{i-1}(\mathrm{~mm})$ & $d_{i}(\mathrm{~mm})$ & $\theta_{i}$ (graus) \\
\hline 1 & 0 & 0 & 0 & $\theta_{1}$ \\
\hline 2 & -90 & 0 & 0 & $\theta_{2}$ \\
\hline 3 & 0 & $a_{2}$ & $d_{3}$ & $\theta_{3}$ \\
\hline 4 & -90 & $a_{3}$ & $d_{4}$ & $\theta_{4}$ \\
\hline 5 & 90 & 0 & 0 & $\theta_{5}$ \\
\hline 6 & -90 & 0 & 0 & $\theta_{6}$ \\
\hline
\end{tabular}

O PUMA 560 é representado na Fig. 2, com os seus seis sistemas de referência fixados aos elos.

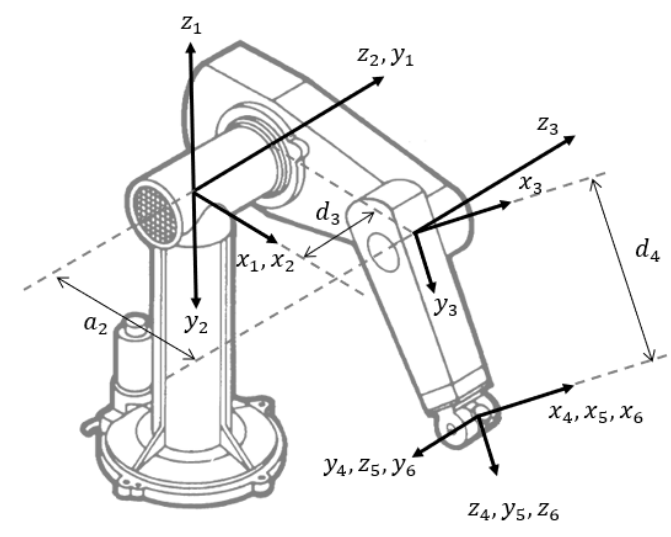

Fig. 2 Sistemas de referência do Puma 560
Após a construção de todas as todas as matrizes de transformação utilizando os parâmetros cinemáticos, e multiplicação das mesmas; obtém-se a matriz de transformação homogênea (9), solução do problema da cinemática direta do PUMA 560.

$$
T=\left[\begin{array}{cccc}
n_{x} & o_{x} & a_{x} & x \\
n_{y} & o_{y} & a_{y} & y \\
n_{z} & o_{z} & a_{z} & z \\
0 & 0 & 0 & 1
\end{array}\right]
$$

Onde:

$$
\begin{gathered}
n_{x}=c_{1}\left(\begin{array}{c}
\left.c_{23}\left(c_{4} c_{5} c_{6}-s_{4} s_{5}\right)-s_{23} s_{5} c_{5}\right) \\
+s_{1}\left(s_{4} c_{5} c_{6}+c_{4} s_{6}\right)
\end{array}\right. \\
n_{y}=s_{1}\left(\begin{array}{c}
\left.c_{23}\left(c_{4} c_{5} c_{6}-s_{4} s_{6}\right)-s_{23} s_{5} c_{6}\right) \\
-c_{1}\left(s_{4} c_{5} c_{6}+c_{4} s_{6}\right)
\end{array}\right. \\
n_{z}=-s_{23}\left(c_{4} c_{5} c_{6}-s_{4} s_{6}\right)-c_{23} s_{5} c_{6} \\
o_{x}=c_{1}\left(c_{23}\left(-c_{4} c_{5} s_{6}-s_{4} c_{6}\right)+s_{23} s_{5} s_{6}\right) \\
+s_{1}\left(c_{4} c_{6}-s_{4} c_{5} s_{6}\right) \\
o_{y}=s_{1}\left(c_{23}\left(-c_{4} c_{5} s_{6}-s_{4} c_{6}\right)+s_{23} s_{5} s_{6}\right) \\
-c_{1}\left(c_{4} c_{6}-s_{4} c_{5} s_{6}\right) \\
o_{z}=-s_{23}\left(-c_{4} c_{5} s_{6}-s_{4} c_{6}\right)+c_{23} s_{5} c_{6} \\
a_{x}=-c_{1}\left(c_{23} c_{4} s_{5}+s_{23} c_{5}\right)-s_{1} s_{4} s_{5} \\
a_{y}=-s_{1}\left(c_{23} c_{4} s_{5}+s_{23} c_{5}\right)+c_{1} s_{4} s_{5} \\
a_{z}=s_{23} c_{4} s_{5}-c_{23} c_{5} \\
y=c_{1}\left(a_{2} c_{2}+a_{3} c_{23}-d_{4} s_{23}\right)-d_{3} s_{1} \\
y=s_{1}\left(a_{2} c_{2}+a_{3} c_{23}-d_{4} s_{23}\right)+d_{3} c_{1} \\
z=-a_{3} s_{23}-a_{2} s_{2}-d_{4} c_{23}
\end{gathered}
$$

utilizando $s_{i j}$ para $\operatorname{sen}\left(\theta_{i}+\theta_{j}\right)$ e $c_{i j}$ para $\cos \left(\theta_{i}+\theta_{j}\right)$.

Segundo Craig (2017), para a solução da cinemática inversa de um manipulador robótico com seis graus de liberdade, têmse 12 equações e 6 incógnitas. No entanto, entre as 9 equações que surgem da porção de matriz rotacional de $T$, somente três são independentes. Estas, somadas às equações da porção de vetor posição de $T$, resultam em seis equações com seis incógnitas. Tais equações são não lineares, transcendentais, que podem ser bastante difíceis de serem resolvidas.

A abordagem sistemática para resolver as equações cinemáticas, proposta por Paul (1981), reescreve as equações cinemáticas diretas de várias maneiras diferentes. Desse modo, ambos os lados da equação matricial da cinemática direta são pré e pós-multiplicados por matrizes de transformação inversa. Ao fazer isso, são produzidas equações equivalentes que possuem as mesmas soluções. Cada equação matricial fornece 12 equações não-lineares provenientes das três principais linhas da matriz.

O principal problema é encontrar, neste universo, equações adequadas que possam ser resolvidas analiticamente. 
A solução da cinemática inversa deste manipulador robótico, determinada por Craig (2017) e Ghosal (2006), é dada por (22) a (27).

$$
\begin{aligned}
& \theta_{1}=\operatorname{Atan} 2(y, x)-\operatorname{Atan} 2\left(d_{3}, \pm \sqrt{x^{2}+y^{2}-d_{3}^{2}}\right) \\
& \theta_{3}=\operatorname{Atan} 2\left(a_{3}, d_{4}\right)-\operatorname{Atan} 2\left(K, \pm \sqrt{a_{3}{ }^{2}+d_{4}{ }^{2}-(K)^{2}}\right) ; \\
& K=\frac{\left(x^{2}+y^{2}+z^{2}-a_{2}{ }^{2}-a_{3}{ }^{2}-d_{3}{ }^{2}-d_{4}{ }^{2}\right)}{2 a_{2}} \\
& \theta_{2}=\operatorname{Atan} 2\left(\begin{array}{c}
\left(-a_{3}-a_{2} c_{3}\right) z-\left(c_{1} x+s_{1} y\right)\left(d_{4}-a_{2} s_{3}\right), \\
\left(a_{2} s_{3}-d_{4}\right) z-\left(a_{3}+a_{2} c_{3}\right)\left(c_{1} x+s_{1} y\right)
\end{array}\right)-\theta_{3} \\
& \theta_{4}=\operatorname{Atan} 2\left(\begin{array}{c}
-a_{x} s_{1}+a_{y} c_{1},-a_{x} c_{1}\left(c_{2} c_{3}-s_{2} s_{3}\right) \\
-a_{y} s_{1}\left(c_{2} c_{3}-s_{2} s_{3}\right)+a_{z}\left(c_{2} s_{3}+s_{2} c_{3}\right)
\end{array}\right) \\
& \theta_{5}=\operatorname{Atan} 2\left(\begin{array}{c}
-\left(\begin{array}{c}
a_{x}\left(c_{1}\left(c_{2} c_{3}-s_{2} s_{3}\right) c_{4}+s_{1} s_{4}\right)+ \\
a_{y}\left(s_{1}\left(c_{2} c_{3}-s_{2} s_{3}\right) c_{4}-c_{1} s_{4}\right)- \\
a_{z}\left(\left(c_{2} s_{3}+s_{2} c_{3}\right) c_{4}\right) \\
a_{x}\left(-c_{1}\left(c_{2} s_{3}+s_{2} c_{3}\right)\right)+ \\
a_{y}\left(-s_{1}\left(c_{2} s_{3}+s_{2} c_{3}\right)\right) \\
+a_{z}\left(-\left(c_{2} c_{3}-s_{2} s_{3}\right)\right)
\end{array}\right)
\end{array}\right)
\end{aligned}
$$

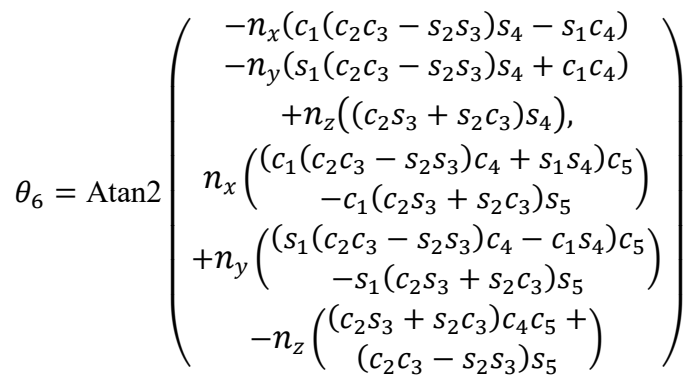

Com o objetivo de mensurar o custo computacional para determinar todas as configurações de junta para posicionar e orientar o efetuador arbitrariamente no espaço, os seguintes valores de junta: $\theta=[0,0,0,0,0,0]$, e demais parâmetros nãonulos: $a_{2}=100, a_{3}=80, d_{3}=50$ e $d_{4}=40$ foram substituídos em (9), desta forma, obtém-se (28).

$$
T=\left[\begin{array}{cccc}
1 & 0 & 0 & 180 \\
0 & -1 & 0 & 50 \\
0 & 0 & -1 & -40 \\
0 & 0 & 0 & 1
\end{array}\right]
$$

A partir deste ponto, as equações (10) a (21) são reescritas substituindo $n_{x}, n_{y}, n_{z}, o_{x}, o_{y}, o_{z}, a_{x}, a_{y}, a_{z}, x, y$ e $z$ pelos respectivos valores da matriz numérica obtida.

Utiliza-se o comando Solve do Maple para encontrar todos os valores de $\theta=\left[\theta_{1}, \theta_{2}, \theta_{3}, \theta_{4}, \theta_{5}, \theta_{6}\right]$, que permitam ao robô assumir a configuração (posição + orientação) dada por (28).

Em 824 milésimos de segundo, consumindo 100,12 MB de memória, e realizando 42512 operações de ponto flutuante (flop), obtém-se oito configurações de junta distintas, conforme pode-se observar na Tabela 3.
Tabela 3. Configurações de juntas do Puma 560 obtidas a partir do método de Paul

\begin{tabular}{|c|c|c|c|c|c|}
\hline $\begin{array}{c}\theta_{1} \\
\text { (graus) }\end{array}$ & $\begin{array}{c}\theta_{2} \\
\text { (graus) }\end{array}$ & $\begin{array}{c}\theta_{3} \\
\text { (graus) }\end{array}$ & $\begin{array}{c}\theta_{4} \\
\text { (graus) }\end{array}$ & $\begin{array}{c}\theta_{5} \\
\text { (graus) }\end{array}$ & $\begin{array}{c}\theta_{6} \\
\text { (graus) }\end{array}$ \\
\hline 0 & 0 & 0 & 0 & 0 & 0 \\
\hline 0 & 0 & 0 & 180 & 0 & 180 \\
\hline 0 & 25,058 & $-53,129$ & 0 & 28,073 & 0 \\
\hline 0 & 25,058 & $-53,129$ & 180 & $-28,073$ & 180 \\
\hline$-148,95$ & 180 & $-53,129$ & 0 & $-126,87$ & $-148,95$ \\
\hline$-148,95$ & 180 & $-53,129$ & 180 & $-126,87$ & 31,048 \\
\hline$-148,95$ & 154,94 & 0 & 0 & $-154,94$ & $-148,95$ \\
\hline$-148,95$ & 154,94 & 0 & 180 & 154,94 & 31,048 \\
\hline
\end{tabular}

Este artigo comprovará que ao utilizar a Teoria de Bases de Gröbner, o custo computacional para determinar a solução da cinemática inversa de manipuladores seriais de cadeia aberta é reduzido consideravelmente.

\section{CINEMÁTICA INVERSA A PARTIR DA TEORIA DAS BASES DE GRÖBNER}

O problema da cinemática inversa consiste em determinar todas as combinações de configurações de juntas que posicionarão o efetuador final do robô em um determinado ponto no espaço (Craig, 2017). Para resolver este problema, deve-se determinar equações polinomiais que modelem o movimento do braço robótico em cada configuração de junta, de modo que o seu efetuador possa ser posicionado em um determinado ponto no espaço. Com todos estes elementos, deve-se encontrar um conjunto de soluções a partir dos possíveis movimentos de cada junta.

\subsection{Cinemática Inversa do SCARA Stäubli TS20 a partir da Teoria das Bases de Gröbner}

Tomando uma vista superior do robô SCARA Stäubli TS20, pode-se projetar os movimentos das duas variáveis de junta rotativas no plano $x y$, utilizando o ponto $(x ; y)$ para representar o efetuador final no espaço. Para resolver o problema da cinemática inversa, estas equações polinomiais devem necessariamente descrever o comportamento dos ângulos de junta $\theta_{1}$ e $\theta_{3}$ em termos do ponto $(x ; y)$ conforme pode-se observar na Fig. 3 .

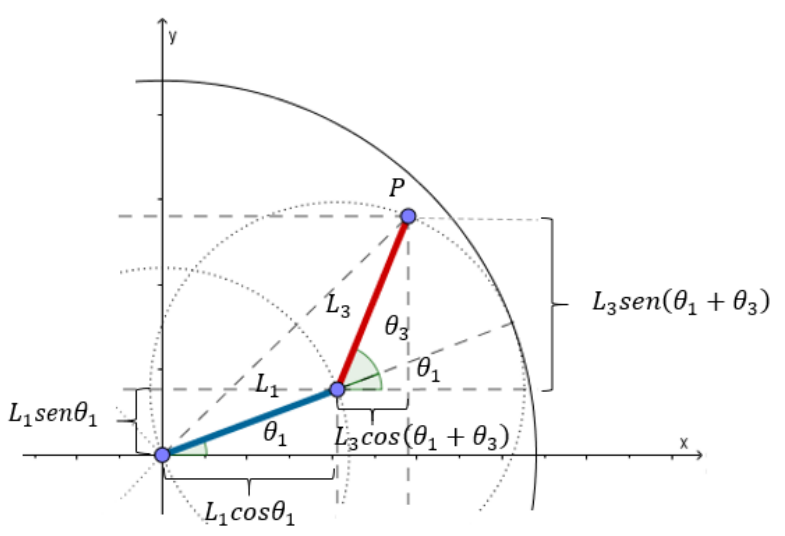

Fig. 3 Modelo algébrico do SCARA Stäubli TS20 
Pela geométrica do manipulador robótico, observa-se que a segunda junta desloca-se na direção do eixo $z$, desta forma é possível determinar imediatamente o valor desta variável de junta prismática como: $d_{2}=z-347$, sendo desnecessário a inclusão desta equação no modelo algébrico do robô.

Fazendo a análise geométrica do modelo e considerando os comprimentos dos elos do robô manipulador $L_{1}=L_{3}=$ $110 \mathrm{~mm}$, são deduzidas as equações: $(x-m, y-n)=$ $\left(110 c_{13}, 110 s_{13}\right)$ e $(m, n)=\left(110 c_{1}, 110 s_{1}\right)$.

Fazendo as devidas substituições e sabendo-se que a soma dos quadrados do seno e cosseno do mesmo ângulo sempre será igual a 1 , determinam-se as equações: $110\left(c_{1} c_{3}-s_{1} s_{3}\right)+$ $110 c_{1}-x=0,110\left(s_{1} c_{3}+s_{3} c_{1}\right)+110 s_{1}-y=0, c_{1}{ }^{2}+$ $s_{1}{ }^{2}-1=0$ e $c_{3}{ }^{2}+s_{3}{ }^{2}-1=0$.

Usando a ordem lexicográfica $c_{3}>s_{3}>c_{1}>s_{1}$ (com o objetivo de isolar inicialmente $s_{1}$ ) e as equações: $f_{1}=$ $L_{3}\left(c_{1} c_{3}-s_{1} s_{3}\right)+L_{1} c_{1}-x, f_{2}=L_{3}\left(s_{1} c_{3}+s_{3} c_{1}\right)+L_{1} s_{1}-$ $y, f_{3}=c_{1}{ }^{2}+s_{1}{ }^{2}-1, f_{4}=c_{3}{ }^{2}+s_{3}{ }^{2}-1$, considerando que $c_{3}, s_{3}, c_{1}, s_{1}$ atuam como variáveis, $x, y$ como coeficientes e substituindo $L_{1}$ e $L_{3}$ pelos valores dos comprimentos dos elos do robô, utiliza-se o seguinte código no Maple:

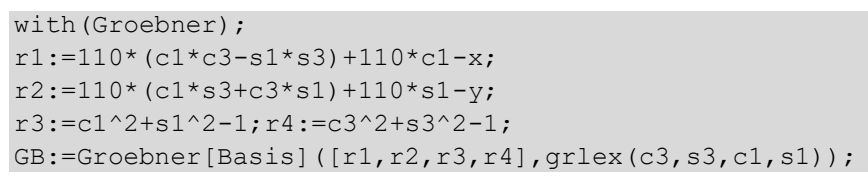

Obtém-se a seguinte base:

$\mathrm{GB}:=\left[\left(48400 * \mathrm{x}^{\wedge} 2+48400 * \mathrm{y}^{\wedge} 2\right){ }^{\star} \mathrm{s} 1^{\wedge} 2+\left(-440 * \mathrm{x}^{\wedge} 2 * \mathrm{y}-0 * \mathrm{y}^{\wedge} 3\right){ }^{\star} \mathrm{s} 1+\right.$ $\left(x^{\wedge} 4+2{ }^{\star} x^{\wedge} 2 * y^{\wedge} 2-48400 * x^{\wedge} 2+y^{\wedge} 4\right),(220 * x) * c 1+(220 * y) * s 1+$ $\left(-x^{\wedge} 2-y^{\wedge} 2\right), 110 * s 3+(-y){ }^{*} c 1+(x) * s 1,110 * c 3+110 * c 1 \wedge 2+(-x) *$ $\mathrm{c} 1+110 * \mathrm{~s} 1 \wedge 2+(-\mathrm{y}) * \mathrm{~s} 1]$

Com o objetivo de determinar todos os valores de $\theta_{1}$ e $\theta_{3}$, que permitam ao robô posicionar o seu efetuador no ponto $(55+55 \sqrt{3} ; 55+55 \sqrt{3})$, escolhido arbitrariamente; utiliza-se o comando Solve do Maple:

\section{with (linalg)}

sys_1:= $110 * \sin ($ theta 3$)-(55+55 * \operatorname{sqrt}(3)) * \cos ($ theta 1$)+$ $(55+55 * \operatorname{sqrt}(3)) * \sin (\operatorname{theta} 1), 110 * \cos ($ theta 3$)+110 *$ $\cos ($ theta 1$) \wedge 2-(55+55 * \operatorname{sqrt}(3)) * \cos ($ theta 1$)+110 *$ $\sin ($ theta 1$) \wedge 2-(55+55 * \operatorname{sqrt}(3)) * \sin ($ theta 1$),(220 *(55+$ $55 * \operatorname{sqrt}(3))) * \cos (\operatorname{theta} 1)+(220 *(55+55 * \operatorname{sqrt}(3)))$ * $\sin ($ theta 1$)-\left(55+55^{*} \operatorname{sqrt}(3)\right)^{\wedge} 2-\left(55+55^{*} \operatorname{sqrt}(3)\right)^{\wedge} 2$, $\left(48400 *\left(55+55^{*} \operatorname{sqrt}(3)\right)^{\wedge} 2+48400 *\left(55+55^{\star} \operatorname{sqrt}(3)\right)^{\wedge} 2\right)$ * $\sin (\text { theta } 1)^{\wedge} 2+\left(-440 *(55+55 * \operatorname{sqrt}(3))^{\wedge} 2 *(55+\right.$ $55 * \operatorname{sqrt}(3))-440 *(55+55 * \operatorname{sqrt}(3)) \wedge 3) * \sin ($ theta 1$)+(55+$ $55 * \operatorname{sqrt}(3))^{\wedge} 4+2 *(55+55 * \operatorname{sqrt}(3))^{\wedge} 2 *\left((55+55 * \operatorname{sqrt}(3))^{\wedge} 2\right)$ $\left.-48400 *\left(55+55^{\star} \operatorname{sqrt}(3)\right)^{\wedge} 2+\left(55+55^{*} \operatorname{sqrt}(3)\right)^{\wedge} 4\right\}$

solve (sys_1, \{theta1, theta3\})

Obtém-se duas configurações distintas para $\theta_{1}$ e $\theta_{3}$ :

$\{$ theta $1=(1 / 6) * P i$, theta $3=(1 / 6) * P i\}$, $\{$ theta $1=(1 / 3) * P i$, theta $3=-(1 / 6) * P i\}$

Os quatro polinômios da base de Gröbner gerada nesta seção, produzem as mesmas soluções que às obtidas a partir das equações (7) e (8). Pode-se comprovar essa afirmação, substituindo os valores de $x$ e $y$ nas equações deduzidas na seção 3.1 deste artigo.

Ao analisar o primeiro elemento na base gerada, pode-se concluir que este elemento é um polinômio de segundo grau em termos de $s_{1}$. Examinando o discriminante desta equação, haverá duas soluções reais distintas para $s_{1}$ quando: $-193600 x^{2}\left(x^{2}+y^{2}\right)\left(x^{2}+y^{2}-48400\right)>0$, e para cada um dos valores obtidos haverá um valor correspondente para $c_{1}$. Como $s_{3}$ depende de $s_{1}$, haverá duas soluções reais para $\theta_{3}$ quando $\theta_{1}$ assumir dois valores distintos.

A partir do gráfico desta inequação, conclui-se que a região descrita é o interior de uma circunferência de raio 220. Considerando os limites angulares impostos pela geometria do SCARA Stäubli TS20, $-133^{\circ}<\theta_{1}<133^{\circ}$ e $-131^{\circ}<$ $\theta_{3}<131^{\circ}$, pode-se observar na Fig. 4 que a área determinada a partir de um dos polinômios da Base de Gröbner, coincide com o volume de trabalho do robô.

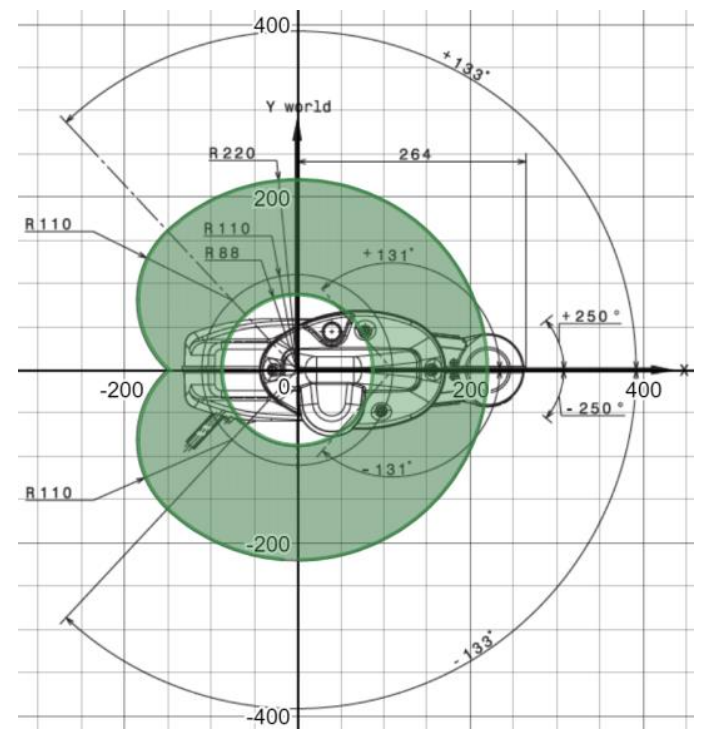

Fig. 4 Volume de trabalho do SCARA Stäubli TS20

Desta forma, ao calcular uma base de Gröbner para um robô serial do tipo SCARA, é possível determinar quais pontos, dentro do espaço de trabalho, têm duas soluções (duas configurações elo/junta), quais pontos têm uma solução (uma configuração elo/junta) e quais pontos não têm solução.

\subsection{Cinemática Inversa do Unimation PUMA 560 a partir da Teoria das Bases de Gröbner}

Para o manipulador PUMA 560, pretende-se determinar todas as configurações de junta para posicionar e orientar o efetuador do manipulador robótico no espaço, essência da cinemática inversa. Para atender a este objetivo, são substituídos arbitrariamente os seguintes valores de junta: $\theta=[0,0,0,0,0,0]$, e demais parâmetros não-nulos: $a_{2}=100$, $a_{3}=80, d_{3}=50$ e $d_{4}=40$ em (9), exatamente conforme realizado na seção 3.2 .

A partir deste ponto, as equações (10) a (21) são reescritas substituindo $n_{x}, n_{y}, n_{z}, o_{x}, o_{y}, o_{z}, a_{x}, a_{y}, a_{z}, x, y$ e $z$ pelos respectivos valores da matriz numérica obtida, exatamente igual à matriz (28). Todos esses 12 polinômios, adicionados aos 6 polinômios obtidos a partir da relação trigonométrica fundamental para cada variável de junta, são utilizados para gerar uma base de Gröbner. O código utilizado no Maple para gerar a base encontra-se no Apêndice A deste artigo. 
Foram utilizados os mesmos valores atribuídos arbitrariamente na seção 3.2, com o objetivo de comparar a eficiência computacional para solução do problema da cinemática inversa utilizando o método de Paul a partir da matriz de Denavit-Hartenberg e a Teoria das Bases de Gröbner para o mesmo manipulador 6R.

Em 31 milésimos de segundo, consumindo 37,18 MB de memória, e realizando 928 operações de ponto flutuante (flop), obtém-se a seguinte base de Gröbner contendo 13 polinômios.

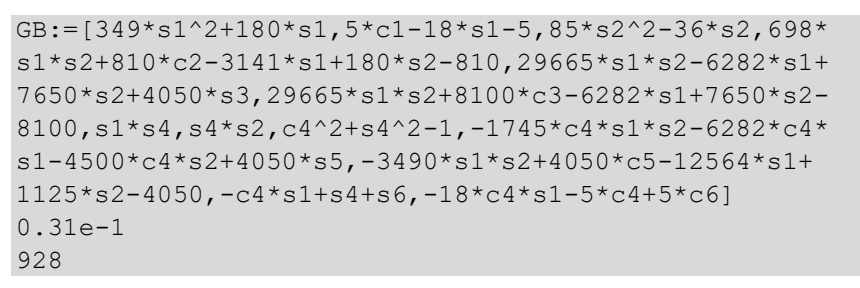

Utiliza-se o comando Solve do Maple para encontrar todos os valores de $\theta=\left[\theta_{1}, \theta_{2}, \theta_{3}, \theta_{4}, \theta_{5}, \theta_{6}\right]$, que permitam ao robô assumir a configuração (posição + orientação) dada por (28).

O código utilizado no Maple para solucionar o sistema de equações, bem como a solução do mesmo, encontram-se no Apêndice B deste trabalho.

Em 531 milésimos de segundo, consumindo 58,19 MB de memória, e realizando 20614 operações de ponto flutuante (flop), obtém-se oito configurações de junta distintas, conforme pode-se observar na Tabela 4.

Tabela 4. Configurações de juntas do Puma 560 obtidas a partir da Teoria das Bases de Gröbner

\begin{tabular}{|c|c|c|c|c|c|}
\hline $\begin{array}{c}\theta_{1} \\
\text { (graus) }\end{array}$ & $\begin{array}{c}\theta_{2} \\
\text { (graus) }\end{array}$ & $\begin{array}{c}\theta_{3} \\
\text { (graus) }\end{array}$ & $\begin{array}{c}\theta_{4} \\
\text { (graus) }\end{array}$ & $\begin{array}{c}\theta_{5} \\
\text { (graus) }\end{array}$ & $\begin{array}{c}\theta_{6} \\
\text { (graus) }\end{array}$ \\
\hline$-148,95$ & 180 & $-53,129$ & 0 & $-126,87$ & $-148,95$ \\
\hline$-148,95$ & 180 & $-53,129$ & 180 & $-126,87$ & 31,048 \\
\hline 0 & 0 & 0 & 0 & 0 & 0 \\
\hline$-148,95$ & 154,94 & 0 & 0 & $-154,94$ & $-148,95$ \\
\hline$-148,95$ & 154,94 & 0 & 180 & 154,94 & 31,048 \\
\hline 0 & 25,058 & $-53,129$ & 0 & 28,073 & 0 \\
\hline 0 & 25,058 & $-53,129$ & 180 & $-28,073$ & 180 \\
\hline 0 & 0 & 0 & 180 & 0 & 180 \\
\hline
\end{tabular}

Vale salientar que todas as equações apresentadas na Seção 3, utilizando o método de Paul para solução da cinemática inversa do manipulador PUMA 560, foram calculadas pelo Maple em 824 milésimos de segundo, consumindo 100,12 MB de memória, e realizando 42512 operações de ponto flutuante para determinar as oito configurações distintas, conforme mostradas na Tabela 3, exatamente iguais às configurações determinadas nesta seção.

Adicionando as 928 operações de ponto flutuante, os 31 milésimos de segundo de tempo de execução e o consumo de 37.18 MB de memória para obtenção da base de Gröbner, ao número de operações de ponto flutuante, tempo de execução e quantidade de memória consumida para obtenção das oito configurações de junta distintas a partir da base de Gröbner gerada; obtém-se 21542 operações de ponto flutuante, 562 milésimos de segundo e 95.37 MB de memória para execução dos dois processos.
Pode-se observar na Tabela 5 que todos os valores mensurados, são inferiores aos obtidos pelo método de Paul a partir da matriz obtida pelo algoritmo de Denavit-Hartenberg.

Tabela 5. Dados de Desempenho Computacional

\begin{tabular}{|c|c|c|}
\hline Dados & $\begin{array}{c}\text { Cinemática } \\
\text { Inversa a partir do } \\
\text { método de Paul }\end{array}$ & $\begin{array}{c}\text { Cinemática Inversa a } \\
\text { partir da Teoria das } \\
\text { Bases de Gröbner }\end{array}$ \\
\hline $\begin{array}{c}\text { Tempo de } \\
\text { execução }\end{array}$ & $824 \mathrm{~ms}$ & $562 \mathrm{~ms}$ \\
\hline Memória & $100,12 \mathrm{MB}$ & $95,37 \mathrm{MB}$ \\
\hline $\begin{array}{c}\text { Operações } \\
\text { de ponto } \\
\text { flutuante }\end{array}$ & $42512 \mathrm{flop}$ & $21542 \mathrm{flop}$ \\
\hline
\end{tabular}

\section{CONCLUSÕES}

Ao calcular uma base de Gröbner, é possível determinar os pontos que podem ser alcançados dentro do volume de trabalho de um manipulador robótico serial do tipo SCARA.

Ao utilizar o método das Bases de Gröbner para a solução da cinemática inversa de manipuladores 6R, observa-se claramente nos testes realizados durante esta pesquisa, que o mesmo se mostrou computacionalmente mais eficiente que o método de Paul, uma vez que as equações produzidas para determinação das seis variáveis de junta são matematicamente mais simples de serem resolvidas, uma vez que através de substituições retroativas, pode-se determinar todas as soluções de configurações de junta para se posicionar e orientar o efetuador do manipulador robótico no espaço.

De acordo com os dados de desempenho obtidos, pode-se afirmar que o método proposto neste trabalho para a solução da cinemática inversa de um robô manipulador 6R é aproximadamente duas vezes mais eficiente computacionalmente que o método de Paul.

Desta forma, entende-se evidenciar a razão pela qual este método é mais eficiente para esta família de manipuladores robóticos.

\section{REFERÊNCIAS}

Boas, A.A.V. and Marcos, E.N. (2016) Uma Introdução A Teoria De Bases De Gröbner Para Algebras Associativas, Livraria da Física.

Buchberger, B. (1995) 'Gröbner Bases: An Algorithmic Method in Polynomial Ideal Theory', in Bose, N.K. (ed.) Multidimensional Systems Theory and Applications, Springer Netherlands.

Buchberger, B. (2006) 'Bruno Buchberger's PhD thesis 1965: An algorithm for finding the basis elements of the residue class ring of a zero dimensional polynomial ideal', Journal of Symbolic Computation, vol. 41, pp. 475-511.

Cox, D., Little, and O'Shea, D. (1992) Ideals, Varieties, and Logarithms: An Introduction to Computational Algebraic Geometry and Commutative Algebra, 4th edition, New York: Springer. 
Craig, J.J. (2017) Introduction To Robotics: Mechanics And Control, 4th edition, Boston: Addison-Wesley.

Denavit, J. and Hartenberg, R.S. (1955) Description and displacement analysis of mechanics based on the $2 \times 2$ dual matrices, Evanston: University of Northwestern.

Duffy, J. and Crane, C.D. (1980) 'Displacement analysis of the general 7-Link 7R mechanism', Mechanism and Machine Theory, vol. XV, pp. 153-169.

Ghosal, A. (2006) Robotics: Fundamental Concepts and Analysis, New Delhi: Oxford University Press.

Hartenberg, R.S. and Denavit, J. (1965) Kinematic synthesis of linkages. Col: McGraw-Hill series in mechanical engineering, New York: McGraw-Hill.

Huang, B. and Milenkovic, V. (1983) 'Kinematics of Major Robot Linkages', Robotics International of SME 2, 1631.

Lee, C.S.G. (1982) 'Robot Arm Kinematics, Dynamics, and Control', Computer, pp. 62-80.

Manseur, R. and Doty, K.L. (1988) 'A Fast Algorithm for Inverse Kinematic Analysis of Robot Manipulators', The International Journal of Robotics Research.

Paul, R.P. (1981) Robot manipulators: mathematics, programming, and control : the computer control of robot manipulators, Cambridge, MA: MIT Press.

Paul, R.P., Shimano, B. and Mayer, G., (1981) 'Kinematic control equations for simple manipulators', Tutorial on Robotics.

Robbiano, L. (1998) 'Groebner Bases and Statistics', in Buchberger, B. and Winkler, F. (ed.) Groebner Bases and Applications, Cambridge University Press.

Sciavicco, L. and Siciliano, B. (1996) Modeling and control of robot manipulators, New York: Mc Graw-Hill.

Sturmfels, B. (2005) 'What is... a Gröbner Basis?', Notices of the American Mathematical Society, vol. 52, no. 10, pp. 1199-1200.

Wang, D. (1998) 'Groebner Bases Applied to Geometric Theorem Proving and Discovering', in Press, C.U. (ed.) Groebner Bases and Applications, Buchberger, Bruno; Winkler, Franz edition.

Wang, Y., Hang, L. and Yang, T. (2006) 'Inverse Kinematics Analysis of General 6R Serial Robot Mechanism Based on Groebner Base', Frontiers of Mechanical Engineering in China, vol. I, January, pp. 115-124.

Yih, T.C. and Youm, Y. (1988) 'Geometrical Modeling of Lower-Pairs Based on Spherical-Euler Geometry', Trends and Developments in Mechanisms, Machines, and Robotics.

\section{Apêndice A. CÓDIGO MAPLE PARA GERAR A BASE DE GRÖBNER DO PUMA 560}

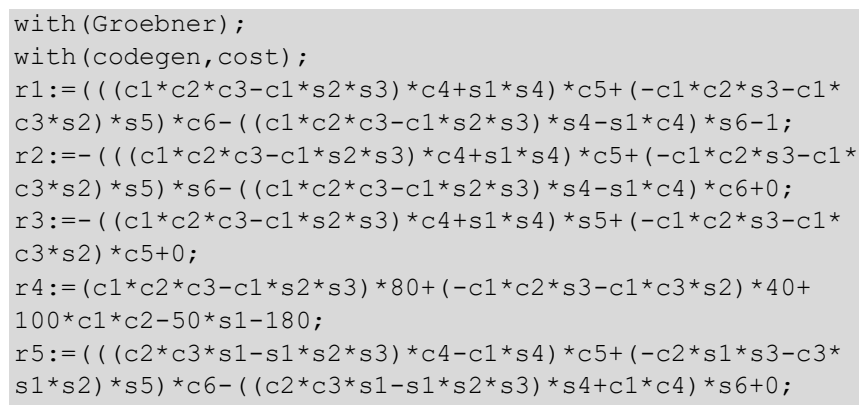

$r 6:=-(((c 2 * c 3 * s 1-s 1 * s 2 * s 3) * c 4-c 1 * s 4) * c 5+(-c 2 * s 1 * s 3-c 3 *$ $\left.\left.\mathrm{s} 1{ }^{*} \mathrm{~s} 2\right){ }^{*} \mathrm{~s} 5\right){ }^{*} \mathrm{~s} 6-\left(\left(\mathrm{c} 2{ }^{*} \mathrm{c} 3{ }^{*} \mathrm{~s} 1-\mathrm{s} 1{ }^{*} \mathrm{~s} 2{ }^{*} \mathrm{~s} 3\right){ }^{*} \mathrm{~s} 4+\mathrm{c} 1{ }^{*} \mathrm{c} 4\right){ }^{*} \mathrm{c} 6+1$; $r 7:=-((c 2 * c 3 * s 1-s 1 * s 2 * s 3) * c 4-c 1 * s 4) * s 5+(-c 2 * s 1 * s 3-c 3 *$ $\left.\mathrm{s} 1{ }^{*} \mathrm{~s} 2\right){ }^{*} \mathrm{C} 5+0$;

$r 8:=(c 2 * c 3 * s 1-s 1 * s 2 * s 3) * 80+(-c 2 * s 1 * s 3-c 3 * s 1 * s 2) * 40+$ $100 *$

$\mathrm{s} 1{ }^{*} \mathrm{C} 2+50 * \mathrm{C} 1-50$;

$r 9:=\left(\left(-c 2{ }^{*} s 3-c{ }^{*} s 2\right) * c 4 * c 5+\left(-c 2{ }^{*} c 3+s 2 * s 3\right) * s 5\right) * c 6-(-c 2 *$ $\mathrm{s} 3-\mathrm{c} 3 * \mathrm{~s} 2) * \mathrm{~s} 4 * \mathrm{~s} 6+0$;

$r 10:=-((-c 2 * s 3-c 3 * s 2) * c 4 * c 5+(-c 2 * c 3+s 2 * s 3) * s 5) * s 6-(-c 2$ *s $3-c 3 * s 2) * s 4 * c 6+0$;

$r 11:=-(-c 2 * s 3-c 3 * s 2) * c 4 * s 5+(-c 2 * c 3+s 2 * s 3) * c 5+1$;

$r 12:=(-c 2 * s 3-c 3 * s 2) * 80+(-c 2 * c 3+s 2 * s 3) * 40-100 * s 2+40$;

$\mathrm{r} 13:=\mathrm{c} 1^{\wedge} 2+\mathrm{s} 1^{\wedge} 2-1$;

$\mathrm{r} 14:=\mathrm{c} 2^{\wedge} 2+\mathrm{s} 2^{\wedge} 2-1$

$r 15:=\mathrm{c} 3^{\wedge} 2+\mathrm{s} 3^{\wedge} 2-1$

$\mathrm{r} 16:=\mathrm{c} 4^{\wedge} 2+\mathrm{s} 4^{\wedge} 2-1$;

$\mathrm{r} 17:=\mathrm{c} 5^{\wedge} 2+\mathrm{s} 5^{\wedge} 2-1$;

$r 18:=c 6^{\wedge} 2+s 6^{\wedge} 2-1$;

$\mathrm{t}:=$ time () ;

$\mathrm{GB}:=$ Groebner [Basis $]$ ( $[r 1, r 2, r 3, r 4, r 5, r 6, r 7, r 8, r 9, r 10$, $r 11, r 12, r 13, r 14, r 15, r 16, r 17, r 18], p l e x(c 6, s 6, c 5, s 5, c 4$, $\mathrm{s} 4, \mathrm{c} 3, \mathrm{~s} 3, \mathrm{c} 2, \mathrm{~s} 2, \mathrm{c} 1, \mathrm{~s} 1))$;

time () -t;

cost (GB) ;

\section{Apêndice B. CÓDIGO MAPLE PARA DETERMINAÇÃO DAS SOLUÇÕES PARA O SISTEMA DE EQUAÇÕES DA BASE DE GRÖBNER}

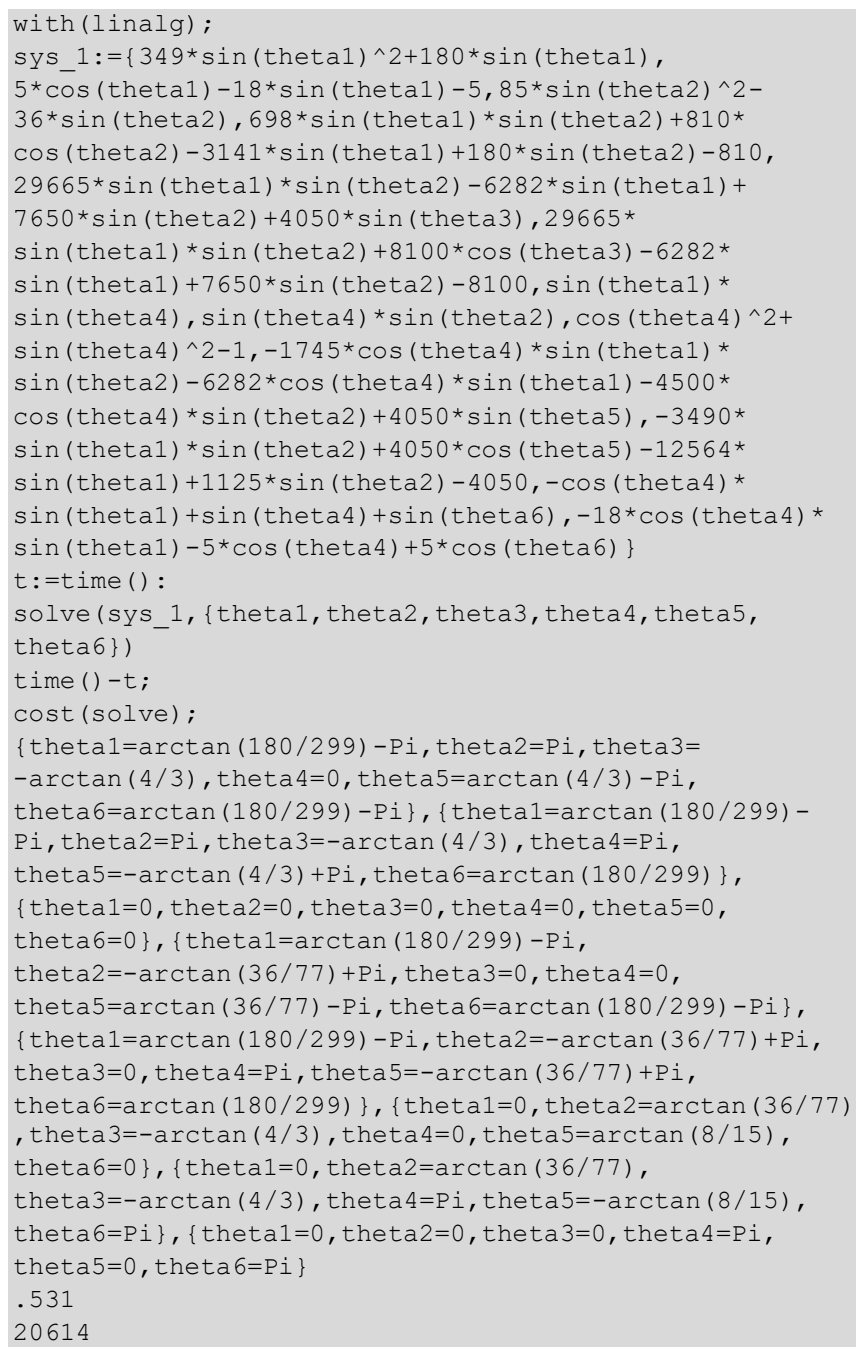

